

\section{Señales Biológicas Cerebrales y Cognición - Biological Brain Signals and Cognition}

Tatiana Castañeda Quirama

Universidad San Buenaventura-Politécnico Grancolombiano

Colombia-Medellín - tatiana.castanedaq@gmail.com

Edisón Ocampo Osorio

Universidad San Buenaventura, Colombia-Medellín

\section{Abstract}

Brain electrical signals allow the brain mechanisms underlying cognitive processes to be subjected to analysis. The present literature review aims to present the results of the most recent studies on brain signals involved in cognition. The literature search was performed using databases such as Google Scholar, Jama Neurology and PubMed. Twelve studies conducted between 2015 and 2020 were selected. The findings report that the processing of brain electrical signals is variable and that such variability is linked to the specific demands and difficulty levels of cognitive tasks.

Keywords: brain signals, cognition, electroencephalogram, empirical/analytical studies. 


\section{Resumen}

Las señales eléctricas cerebrales permiten someter a un análisis, los mecanismos cerebrales que subyacen a los procesos cognitivos. La presente revisión de literatura tiene como objetivo exponer los resultados de los estudios más recientes acerca de las señales cerebrales implicadas en la cognición. La búsqueda bibliográfica se realizó a partir de bases de datos como Google Scholar, Jama Neurology y PubMed. Se seleccionaron 12 estudios realizados entre el año 2015 y 2020. Los hallazgos reportan que el procesamiento de las señales eléctricas cerebrales es variable y que dicha variabilidad está vinculada a las demandas específicas y a los niveles de dificultad de las tareas cognitivas.

Palabras clave: señales cerebrales, cognición, electroencefalograma, estudios empíricos/analíticos. 


\section{Introducción}

Las señales eléctricas del cerebro son designadas como ondas cerebrales y se generan en el momento de realizar actividades mentales o cognitivas que son monitoreadas a partir de herramientas como la electroencefalografía. (Ventura, et al., 2020) Dicha actividad eléctrica tiene una medición en microvoltios y dependiendo de la frecuencia de las descargas eléctricas en los grupos de neuronas estas ondas serán diferentes. Estas señales, tienen una clasificación acorde con su frecuencia y potencia. Se conoce como frecuencia al número de ondas cerebrales que pueden registrarse en un segundo y su medición es por ciclos de segundos o Hertzios $(\mathrm{Hz})$, mientras que la potencia da cuenta de la altura de cada onda durante el registro.

Al hablar de señales cerebrales eléctricas, se emplea el concepto de "oscilación" para hacer referencia a las fluctuaciones en el ritmo de los potenciales postsinápticos de un grupo de neuronas o de una región cortical, además del patrón de descarga en el ritmo que tiene el potencial de acción en una neurona o grupo de neuronas. Esta actividad de oscilación permite sincronizar grupos neuronales que pertenecen a una misma área cortical o a áreas que tienen distancia entre sí, e intervienen en acciones motoras o tareas cognitivas.

En función de la frecuencia de las señales cerebrales eléctricas, estas clasifican en cinco tipos a) Delta con una onda lenta de frecuencia de $0.5 \mathrm{a} 4 \mathrm{~Hz}$ están presentes durante el sueño profundo. b) Theta su frecuencia es de 4 a $7 \mathrm{~Hz}$. Se asocia con capacidades como la imaginación, fantasía y creatividad. Se encuentran en el esfuerzo intelectual relacionado con los procesos de aprendizaje. c) Alfa con una frecuencia de 7 a $12 \mathrm{~Hz}$, están vinculadas con estados de tranquilidad y relajamiento. Se registran especialmente durante momentos previos a 
dormirse. d) Beta su frecuencia está comprendida entre 13 y $39 \mathrm{~Hz}$. Estas señales son registradas en estados de atención o vigilia y cuando se está alerta a algún estímulo (plena actividad mental). Son predominantes en actividades que exigen niveles de concentración elevados y e) Gama son señales cerebrales eléctricas de alta frecuencia $40 \mathrm{hz}$ o más (son las más rápidas). Se relacionan con tareas que exigen un alto nivel de procesamiento cognitivo, como por ejemplo la resolución de problemas. (Sciotto \& Niripil, 2018)

El estudio de la actividad cerebral se fundamenta en la recepción de las señales eléctricas cerebrales a través de dispositivos como el electroencefalograma que permitan su posterior amplificación y el análisis de los datos obtenidos. Dichas señales, se obtienen a partir de electrodos que se ubican en la superficie del cráneo y que registran datos de diferentes regiones del cerebro. (Ventura, et al., 2020)

A partir de lo anterior, es importante destacar que la neurociencia cuenta con un adecuado desarrollo de conocimientos y con herramientas tecnológicas que permiten comprender los procesos cerebrales que están implicados en los procesos cognitivos, por lo cual el objetivo del presente trabajo es realizar una revisión de literatura que dé cuenta de los resultados de estudios recientes acerca de las señales cerebrales y la cognición

\section{Metodología}

Se llevó a cabo una revisión narrativa de literatura, a partir de una exploración sistemática de la información. Este tipo de revisión tiene como finalidad explorar, describir y discutir un determinado tema, a través de la búsqueda de literatura especializada. (Zillmer \& Díaz-Medina, 2018). Para la elaboración del presente artículo, se hizo una revisión de investigaciones de corte empírico analítico que abordaron el tema de las señales cerebrales y los procesos cognitivos. 


\section{Criterios de inclusión}

Los artículos se incluían si: (1) la fecha de publicación comprendía entre el año 2015-2021 (2) los artículos estaban en texto completo en idioma español o inglés; (3) los estudios eran de corte empírico analítico; (4) las investigaciones relacionan las señales cerebrales con los procesos cognitivos y (5) los estudios incluían medidas de las señales cerebrales a partir de electroencefalogramas.

\section{Estrategia de búsqueda}

Se buscaron estudios transversales y longitudinales que daban cuenta de la relación entre las señales cerebrales y la cognición, publicados entre el 2015-2021. La extracción de la información se hizo a partir de tres buscadores: Google Scholar, Jama Neurology y PubMed

Tabla 1

Ecuación de búsqueda.

Google Scholar (Señales AND cerebrales AND Cognición OR Signals AND brain AND Cognition)

Jama Neurology (Signals AND brain AND Cognition OR biological AND brain AND signals AND cognition)

PubMed (Signals AND brain AND Cognition OR biological AND brain AND signals AND cognition)

De los 32 artículos encontrados que relacionaban las señales cerebrales con la cognición, se seleccionaron 12 manuscritos que cumplían con los criterios de inclusión. Todos los artículos hacen parte de investigaciones empírico-analíticas.

\section{Resultados}

Armbruster-Genc, et al. (2016) investigaron la variabilidad de la señal cerebral bajo diferentes demandas en las tareas, es decir, la flexibilidad cognitiva frente a estabilidad cognitiva y su efecto en el rendimiento conductual, con el objetivo de 
comprender a profundidad cómo varían las señales cerebrales para la cognición. Para ello, estimaron la variabilidad de la señal BOLD específica de cada condición y examinaron la relación entre la variabilidad de la señal BOLD y el rendimiento conductual, así como la variabilidad conductual en una muestra de 76 sujetos que no tenían antecedentes de enfermedades neurológicas o psiquiátricas.

Los resultados de este estudio evidencian que existe una mayor dificultad, tanto para el cambio de tarea (es decir, la flexibilidad cognitiva) como para la inhibición del distractor (estabilidad cognitiva). Se demostró que el efecto de la variabilidad de las señales conductuales y cerebrales en el rendimiento de la tarea varía en función de las exigencias de estas. Los datos encontrados, indican que los niveles más altos de variabilidad de la señal cerebral conducen a la reducción de las tasas de error, es decir, a una mayor eficacia del rendimiento.

Ahora bien, Curley, et al. (2018) caracterizaron las señales de EEG utilizadas como indicadores de la cognición en pacientes con lesiones cerebrales graves. Se evaluaron 15 sujetos como grupo control y 28 pacientes con lesiones de distinta etiología, localización y extensión.

La evaluación se centró en los aspectos espaciales y temporales de las señales cerebrales y en cómo se comparan con las observadas en controles sanos, y en el impacto de la fluctuación de la excitación. Las respuestas de los pacientes demostraron un rango diverso de frecuencias de notificación en el espectro de potencia, mientras que las respuestas del grupo control demostraron en gran medida una supresión de la potencia espectral alfa y/o beta durante la realización de la tarea. En concreto, los resultados de los pacientes muestran modulación de la potencia de baja frecuencia, una amplia variación espacial en los canales de información, y un sesgo hacia la evidencia del desempeño de tareas específicas. Las características del espectro de potencia del EEG reflejan la presencia de características de baja frecuencia theta y delta 
Así mismo, León-Ariza, et al. (2017) realizaron un estudio con 20 hombres y 19 mujeres sin diagnóstico neurológico, con el propósito de estimar la relación entre la señal electroencefalográfica orbitofrontal y la actividad del sistema nervioso autónomo implicada en los procesos cognitivos. Los resultados, permiten estimar que aquellos estímulos de tipo visual que se relacionan con procesos cognitivos implicados en el aprendizaje generan estimulación en la corteza orbitofrontal, la cual tiene como función realizar procesos de asociación, ubicación en el espacio, contexto o eventos, las respuestas de tipo emocional y la toma de decisiones.

Estos hallazgos, sugieren entonces la existencia de cambios en la actividad electroencefalográfica de la corteza orbitofrontal, donde se evidencia una mayor actividad teta que está vinculada a mayor activación del sistema nervioso simpático y una respuesta menor en el sistema nervioso parasimpático, mientras que en las señales delta no se evidencia alguna variación. Lo anterior, indica que la corteza cerebral orbitofrontal tiene una participación en la regulación de la actividad autonómica durante períodos de vigilia. Este resultado se hace evidente, en el momento de pasar de un estado de reposo a una actividad cognitiva que requiere de observación, concentración y memoria.

Otro estudio con interés en establecer una relación entre las señales cerebrales y tareas cognitivas, fue la realizada por Wang, et al. (2020), quienes diseñaron una investigación que pretendía explorar los posibles mecanismos que subyacen a las diferencias individuales en la experiencia deportiva, midiendo la variabilidad de las señales cerebrales durante una tarea de control cognitivo. Se empleó la tarea de flanqueo, en tanto proporciona un medio excelente para probar el control atencional y ha demostrado sensibilidad relativamente mayor que otros paradigmas atencionales en estudios que examinan la relación deporte-cognición.

Los datos del comportamiento revelaron que, en comparación con los jugadores de deportes estáticos, los jugadores de deportes de intercepción 
mostraban una respuesta más rápida y mayor estabilidad. El análisis de entropía multiescala (MSE) en la señal del EEG en el sitio parietal, reveló que la variabilidad de la señal cerebral era mayor en las tareas incongruentes, en comparación con los ensayos congruentes para ambos grupos. Lo anterior, demostró que la actividad cerebral aumenta en la región parietal inferior, lo cual puede estar asociado a la ampliación de la atención fuera de la fóvea para incluir la periferia (por ejemplo, los flancos), pero disminuye en la región parietal superior durante los ensayos incongruentes, ya que esta región puede estar más implicada en el estrechamiento de la atención (objetivo central). En conjunto, estos procesos complejos en el área parietal podrían contribuir a la modulación de la variabilidad de la señal cerebral.

En esta misma línea de investigación Anzolin, et al. (2017) ejecutaron una investigación con la finalidad de proporcionar descriptores neurofisiológicos basados en las propiedades de los patrones de conectividad, que permitieran caracterizar los diferentes procesos de atención, para lo cual incluyeron medidas de EEG y tarea de flanqueo. Los resultados evidencian que el componente de alerta está relacionado con la activación de la red fronto-parietal en la onda Alfa, que modula el impacto de una señal de advertencia que precede un objetivo. La resolución de conflictos indico mayor cooperación entre las redes Bottom- Up y Top-Down. Los resultados evidencian actividad en la onda beta.

Flores, et al. (2019) se interesaron en evaluar el efecto que tenía realizar 30 minutos de actividad física aeróbica en la actividad neurofisiológica cerebral, mientras se resolvían pruebas que requerían de un proceso de atención selectiva. Se incluyeron 14 sujetos sin alteraciones físicas y cognitivas y los hallazgos reportaron índices de Hurst $<0,5$ registrados en la corteza prefrontal, temporal y occipital antes y después de realizar ejercicio, lo cual es indicativo de que el rango de ondas beta $(13-30 \mathrm{~Hz})$ tienen una actividad no persistente. En conclusión, se registra un incremento del índice de Hurst en la corteza temporal derecha después de realizar actividad física aeróbica y valores similares en la corteza prefrontal y 
occipital en el momento de registrar las mediciones antes y después de pedir a los participantes realizar ejercicio físico.

Otro de los hallazgos de interés es el de Díaz, et al. (2017) quienes compararon la estimación lineal y no lineal de la sincronía y la organización interna de un conjunto de series temporales electroencefalográficas $(E E G)$ registradas durante la realización de tareas cognitivas en 12 pilotos de avión. Se encontró que cuando la estimación de los valores $\mathrm{H}$ de las bandas beta y gamma del cerebro entero aumentan, hay una disminución del número de pares de electrodos altamente correlacionados $(R>0.85)$. Estos resultados indican que durante una tarea mental se produce una desincronización cerebral global para asignar recursos de procesamiento más específicos (locales) para resolver la tarea cognitiva impuesta. Se concluyó que la actividad eléctrica cerebral tiende a desincronizarse y que cada región trabaja de manera independiente en diferentes aspectos que hacen parte de la resolución de un problema, a medida que incrementa el índice de dificultad.

Los resultados anteriormente expuestos, son coherentes con los de Zarjam, et al. (2015) quienes estudiaron las señales de EEG en una muestra de seis hombres mientras resolvían una actividad cognitiva con siete niveles de complejidad (muy fácil a muy difícil) y que implicaba la memoria de trabajo. Los resultados indicaron que la onda delta en la región frontal y occipital presentaron variaciones según el grado de complejidad que tenía la tarea, incrementando los valores de correlación a medida que incrementa la dificultad, lo cual puso en evidencia un aumento significativo de la complejidad de las señales del EEG.

Otro de los estudios que relaciona las señales cerebrales con la cognición, fue el realizado por Ventura, et al (2020) quienes tenían como objetivo establecer una clasificación de las señales neurológicas que se obtenían a partir de un equipo de electroencefalografía, para así establecer cuáles áreas del cerebro se activan al 
momento de ejecutar tareas cognitivas como la toma de decisiones, en estado de vigilia y de tensión negativa.

Los datos obtenidos de las ondas Beta (que representan el estado de alerta máxima) evidencian que sólo el 10\% de los participantes empleó la región frontal, orientada a definir las respuestas óptimas a las tareas. Así mismo, se encontró que en el $60 \%$ de los evaluados hubo una activación de las zonas cerebrales de las regiones temporales, lo cual significa que en el momento de ejecutar las tareas recurren a experiencias previas y a partir de esto emiten sus respuestas. Estos sujetos estuvieron más atentos a lo que ocurría a su alrededor, en tanto prestaron más atención a la información que recibían de otras áreas cerebrales.

Con respecto a procesos cognitivos como la memoria, Euler, et al., (2016), analizaron la señal de EEG en estado de reposo, estableciendo asociaciones entre los resultados que evidenciaron correlaciones temporales de largo alcance, con discrepancias a nivel individual en la memoria de trabajo. Se evaluaron 54 adultos sanos y las correlaciones se establecieron en las cinco bandas del Electroencefalograma, a través del exponente de Hurst. Los resultados indican una asociación inversa entre las relaciones de las ondas delta y theta y los diferentes rangos de memoria operativa, lo cual muestra la pertinencia del empleo de este tipo de medidas en la comprensión del funcionamiento cerebral.

Con relación al lenguaje, cabe destacar los resultados del trabajo de Benitez-Burraco y Murphy (2016), que indican que en niños con Trastorno del Espectro Autista existe un incremento de la frecuencia en las ondas theta y delta, mientras que existe una reducción de las ondas beta, lo cual demostró ser un factor causal de interferencias en los procesos de comprensión lingüística que necesitan de la interconexión de distintas áreas cerebrales. Lo anterior, también explica la limitación en la capacidad de clasificación sintáctica de las frases que hacen parte del discurso y las alteraciones en las bandas cruzadas alfa - gamma, dan cuenta de 
las dificultades en el reconocimiento de palabras, en tanto esto ocurre en fases de poca activación.

Finalmente, Sánchez y Martín (2018) evaluaron 32 sujetos sin ningún tipo de alteración con el objetivo de estimar diferencias en la expresión de ondas cerebrales alfa mediante tareas cognitivas de imaginación de objetos e imaginación de acciones. Mientras se ejecutó la tarea de imaginar objetos, la distribución de las ondas alfa fue mayor en la zona posterior y hubo una evidente simetría y concentración en las zonas parietales superiores izquierda y derecha. Durante la tarea de imaginación de acciones resaltó un contraste entre la actividad anterior y posterior. Se observa una distribución con dispersión en la actividad alfa parietal izquierda superior, la zona central y precentral izquierda. De los datos obtenidos, se infiere que la corteza premotora izquierda, y la zona temporal occipital derecha, incrementaron de manera significativa la actividad alfa, por lo cual disminuyeron su nivel de activación cuando se pasó de la imaginación de objetos a la imaginación de acciones.

\section{Discusión.}

De acuerdo con los resultados obtenidos de la presente revisión de literatura, es posible evidenciar que los avances tecnológicos en electroencefalografía han sido significativos durante los últimos años, en tanto ha puesto a disposición de clínicos e investigadores un dispositivo que permite detectar actividad cerebral como correlato de actividades cognitivas. Los diversos estudios revisados en la construcción de este artículo han demostrado que el procesamiento de las señales eléctricas cerebrales es variable y que dicha variabilidad está vinculada a la dificultad de la tarea cognitiva. (Armbruster-Genç et al., 2016; Díaz et al., 2017; León-Ariza et al., 2017; Wang et al., 2020, Zarjam, et al.,2015) 
Los autores coinciden en señalar que la variabilidad del procesamiento neural es un indicador relevante de la función cerebral y favorece la adaptación dinámica a las demandas cambiantes del entorno (Díaz et al., 2017; Flores et al., 2019; Ventura et al., 2020; Wang, et al., 2020), además los niveles altos de variabilidad facilitan la flexibilidad cognitiva

De manera específica se ha reportado a partir de medidas electrofisiológicas que la actividad teta incrementa durante los procesos de aprendizaje en áreas como la corteza frontal, la cual participa activamente en la regulación de activación autonómica durante un estado de vigilia. Este fenómeno se evidencia cuando se pasa de un estado de reposo a una demanda cognitiva, y coincide con respuestas que requieren del uso de la memoria episódica, en especial cuando la tarea involucra la memoria operativa (Euler et al., 2016; León-Ariza et al., 2017; Zarjam et al., 2015)

Los estudios dirigidos a la evaluación de procesos cognitivos atencionales indican que los tres componentes de la atención son independientes, implican diferentes áreas anatómicas y cada

de ellos tiene una actividad eléctrica oscilatoria y un curso temporal distintos. Las pruebas muestran una fuerte activación de las zonas corticales frontales y parietales y una gran implicación del hemisferio derecho en la alerta, mientras que la red de control ejecutivo aparece distribuida en varias áreas cerebrales (Anzolin et al., 2017; Flores et al., 2019; Wang et al., 2020)

\section{Conclusión}

Las señales cerebrales presentan oscilación dependiendo del estado cognitivo (reposo-acción) las condiciones de la tarea (instrucciones) y los niveles de dificultad, lo cual permite establecer que cada región cerebral trabaja de manera independiente en aspectos que hacen parte de los procesos cognitivos. Es posible 
deducir que la variabilidad del procesamiento neuronal favorece una adaptación dinámica a las demandas cambiantes del entorno, en tanto facilitan la flexibilidad cognitiva y se asocian a una mayor eficacia en el rendimiento.

\section{Referencias}

Anzolin, A., Mattia, D., Toppi, J., Pichiorri, F., Riccio, A., \& Astolfi, L. (2017). Brain connectivity networks at the basis of human attention components: An EEG study. Proceedings of the Annual International Conference of the IEEE Engineering in Medicine and Biology Society, EMBS, 3953-3956. https://doi.org/10.1109/EMBC.2017.8037721

Armbruster-Genç, D. J. N., Ueltzhöffer, K., \& Fiebach, C. J. (2016). Brain signal variability differentially affects cognitive flexibility and cognitive stability. Journal of Neuroscience, 36(14), 3978-3987. https://doi.org/10.1523/JNEUROSCI.2517-14.2016

Artieda, J., Alegre, M., Valencia, M., Urrestarazu, E., Pérez-Alcázar, M., Nicolás, M.J., López Azcárate, J., \& Iriarte, J.. (2009). Oscilaciones cerebrales: papel fisiopatológico y terapéutico en algunas enfermedades neurológicas y psiquiátricas. Anales del Sistema Sanitario de Navarra, 32, 45-60.

Benitez-Burraco, A., \& Murphy, E. (2016). The Oscillopathic Nature of Language Deficits in Autism: From Genes to Language Evolution. Frontiers in Human Neuroscience. doi: 10.3389/fnhum.2016.00120.eCollection 2016.

Curley, W. H., Forgacs, P. B., Voss, H. U., Conte, M. M., \& Schiff, N. D. (2018). Characterization of EEG signals revealing covert cognition in the injured brain. Brain, 141(5), 1404-1421. https://doi.org/10.1093/brain/awy070

Díaz, H., Maureira, F., \& Córdova, F. (2017). Temporal scaling and inter-individual hemispheric asymmetry of chaos estimation from EEG time 
series. Procedia Computer Science, 122, 339-345. https://doi.org/10.1016/j.procs.2017.11.378

Euler, M. J., Wiltshire, T. J., Niermeyer, M. A., \& Butner, J. E. (2016). Working memory performance inversely predicts spontaneous delta and theta-band scaling relations. Brain Research, 1637, 22-33. https://doi.org/10.1016/j.brainres.2016.02.008

Flores, F., Maureira, F., Díaz, H., Navarro, B., Gavotto, O. y Matheu, A. (2019). Efectos de una sesión de ejercicio físico sobre la actividad neurofisiológica durante la resolución de una prueba de atención selectiva. Retos, 36, 390-396.

León-Ariza, H. H., Botero-Rosas, D. A., Sánchez-Jiménez, A., Ramírez-Villada, J. F., \& Acero-Mondragón, E. J. (2017). Cognición, respuesta electroencefalográfica y su relación con la variabilidad de la frecuencia cardíaca. Revista de La Facultad de Medicina, 65(1), 67-72. https://doi.org/10.15446/revfacmed.v65n1.57140

Sánchez, J., Jofré, M., \& Burán, M. (2018). Las ondas alfa del electroencefalograma cuantificado y su relación con la evocación imaginaria de contenidos diferenciados. http://www.ucongreso.edu.ar/wp-content/uploads/2018/10/Las-ondas-alfa-d el-electroencefalograma-cuantificado-y-su-relación-con-la-evocación-imagi naria-de-contenidos-diferenciados.pdf

Sciotto, E. A., \& Niripil, E. B. (2018). Salud En La Educación Ondas Cerebrales, Conciencia Y Cognición. Salud En La Educación, https://www.researchgate.net/publication/326056524_ONDAS_CEREBRAL ES_CONCIENCIA_Y_COGNICION 
Ventura, A. M.; Fernández, H. A.; Reyes, V. A. \& Gómez, V, M. (2020). La toma de decisiones, un análisis de la actividad cerebral directiva: Funcionamiento del cerebro del directivo. Revista Relayn, 4, (3), 114-127.

Wang, C. H., Liang, W. K., \& Moreau, D. (2020). Differential Modulation of Brain Signal Variability During Cognitive Control in Athletes with Different Domains of Expertise. Neuroscience, 425(2020), 267-279. https://doi.org/10.1016/j.neuroscience.2019.11.003

Zarjam, P., Epps, J., Lovell, N. y Chen F. (2012). Characterization of memory load in an arithmetic task using non-linear analysis of EEG signals. Conf Proc IEEE Eng Med Biol Soc, 2012, 3519-3522.

Zillmer, J. G. V., \& Díaz-Medina, B. A. (2018). Revisión Narrativa: elementos que la constituyen y sus potencialidades. Journal of Nursing and Health, 8(1), 2-3. https://doi.org/10.15210/jonah.v8i1.1365 


\section{About the Authors}

Tatiana Castañeda Quirama: Psychologist Universidad Católica Luis Amigó, Master in Clinical Psychology - Universidad del Norte. Doctoral student in psychology at Universidad San Buenaventura. Full-time professor with research functions at Institución Universitaria Politécnico Grancolombiano. Subjects taught: clinical psychology, psychopathology, psychological evaluation, Fundamentals of cognitive behavioral psychology. With experience as principal investigator and co-investigator of different projects on health issues. She is also the author of several publications related to health in different specialized journals.

Edison Ocampo Osorio: Psychologist. Master in neuropsychology. Doctoral student in psychology. San Buenaventura University-Medellin. Teaching experience at Universidad Católica Luis Amigó and Universidad Cooperativa de Colombia in subjects such as learning theories and basic psychological processes. 